\title{
The extreme ultraviolet excess emission in five clusters of galaxies revisited
}

\author{
F. Durret ${ }^{1}$, E. Slezak ${ }^{2}$, R. Lieu ${ }^{3}$, S. Dos Santos ${ }^{1,4}$, and M. Bonamente ${ }^{3}$ \\ 1 Institut d'Astrophysique de Paris, 98bis Bd Arago, 75014 Paris, France \\ 2 Observatoire de la Côte d'Azur, BP 4229, 06304 Nice Cedex 4, France \\ 3 Department of Physics, University of Alabama, Huntsville AL 35899, USA \\ ${ }^{4}$ Department of Physics and Astronomy, University of Leicester, University Road, Leicester LE1 7RH, UK
}

Received 18 February 2002 / Accepted 15 May 2002

\begin{abstract}
Evidence for excess extreme ultraviolet (EUV) emission over a tail of X-ray gas bremsstrahlung emission has been building up recently, but in some cases remains controversial, mostly due to the moderate quality of the EUV data. In order to improve the signal to noise ratio in the EUV, we have performed the wavelet analysis and image reconstructions for five clusters of galaxies observed both at EUV and X-ray energies with the EUVE and ROSAT satellites respectively. The profiles of the EUV and X-ray reconstructed images all differ at a very large confidence level and an EUV excess over a thermal bremsstrahlung tail is detected in all five clusters (Abell 1795, Abell 2199, Abell 4059, Coma and Virgo) up to large radii. These results, coupled with recent XMM-Newton observations, suggest that the EUV excess is probably non thermal in origin.
\end{abstract}

Key words. galaxies: clusters: individual: Abell 1795, Abell 2199, Abell 4059, Virgo, Coma radiation mechanisms: general - X-rays: galaxies: clusters - cosmology: observations

\section{Introduction}

The Extreme Ultraviolet Explorer (EUVE) has detected emission from a few clusters of galaxies in the $\sim 70-200 \mathrm{eV}$ energy range. By order of discovery, these were: Virgo (Lieu et al. 1996b; Berghöfer et al. 2000a), Coma (Lieu et al. 1996a), Abell 1795 (Mittaz et al. 1998), Abell 2199 (Bowyer et al. 1998), Abell 4059 (Berghöfer et al. 2000b) and Fornax (Bowyer et al. 2001). The problem of the physical origin of the EUV emission was immediately raised. Excess EUV emission relative to the extrapolation of the X-ray emission to the EUVE energy range was detected in several clusters, suggesting that thermal bremsstrahlung from the hot $\left(\sim 10^{8} \mathrm{~K}\right)$ gas could not be entirely responsible for the EUV emission. Although there has been some controversy on the reality of this EUV emission, it now seems well established for the following clusters: Coma (Lieu et al. 1996a), Virgo (Lieu et al. 1996b; Berghöfer et al. 2000a; Bonamente et al. 2001a), Abell 1795 (Mittaz et al. 1998; Bonamente et al. 2001a), Abell 2199 (Bowyer et al. 1998; Kaastra et al. 1999; Lieu et al. 1999b) and Abell 4038 (Bowyer et al. 1998).

This excess EUV emission can be interpreted as due to two different mechanisms: thermal radiation from a warm $\left(10^{5}-10^{6} \mathrm{~K}\right)$ gas, as first suggested by Lieu et al. (1996a), or inverse Compton emission of relativistic electrons either on the cosmic microwave background or on stellar light originating

Send offprint requests to: F. Durret, e-mail: durret@iap. fr in galaxies (Hwang 1997; Bowyer \& Berghöfer 1998; Ensslin \& Biermann 1998; Sarazin \& Lieu 1998; Ensslin et al. 1999), or both. Note that although the existence of a multiphase intracluster medium is plausible (Lieu et al. 2000; Bonamente et al. 2001b), there are several difficulties with the thermal model. The most serious one is that since gas in the temperature range $10^{5}-10^{6} \mathrm{~K}$ cools very rapidly, a source of heating for this gas is necessary, and only one model has been proposed to account for this gas heating (Fabian 1997). Besides, the nondetection by the Far Ultraviolet Spectroscopic Explorer of the OVI $\lambda \lambda 1032,1038$ lines in the spectra of the Coma and Virgo clusters could exclude the presence of warm gas at a $2 \sigma$ level (Dixon et al. 2001). On the other hand, non thermal models (Sarazin \& Lieu 1998; Bowyer \& Berghöfer 1998; Lieu et al. 1999a; Ensslin et al. 1999; Atoyan \& Völk 2000; Brunetti et al. 2001) seem able to account for the excess EUV emission, but require very high cosmic ray pressure.

Following a controversy that the extended EUV emission from some clusters could be simply an artefact of bad background subtraction (Bowyer et al. 1999; Lieu et al. 1999b) we have performed a wavelet analysis and reconstruction of the EUVE images of several clusters. Our aims were to confirm the detection of EUV emission as far as possible from the cluster center, to derive accurate EUV and X-ray profiles and to confirm the existence and radial distributions of the soft excesses over thermal bremsstrahlung in these clusters.

We present here the wavelet reconstruction of the images of these five clusters, both in the EUV (EUVE images) and in 
X-rays (ROSAT PSPC images). Abell 4038 was also analyzed but was discarded due to its low signal to noise ratio in the EUV. We then compare the EUV and X-ray profiles of the images thus obtained, arguing that if both emissions have the same physical origin it should not be possible to distinguish the shapes of these profiles. In fact, we will see that they are found to be different in all clusters, and that soft excesses over thermal bremsstrahlung are detected in all five clusters with various radial distributions.

The data and method are presented in Sect. 2. Results are presented in Sect. 3 and discussed in Sect. 4.

\section{The data and image analysis}

\subsection{The data}

Five clusters were observed in the EUV range with the EUVE satellite and in X-rays with the ROSAT PSPC satellite (archive data). The exposure times and main cluster characteristics are given in Table 1.

In order to have comparable spatial resolutions in the EUV and X-rays, the EUV images were rebinned $4 \times 4$, leading to a "superpixel" size of $0.3077 \operatorname{arcmin}(18.5 \mathrm{arcsec})$. X-ray images were also rebinned to the same 18.5 arcsec pixel size in order to allow a direct comparison of the EUV and X-ray profiles.

The linear scale per superpixel at the cluster distance, estimated with $H_{0}=50 \mathrm{~km} \mathrm{~s}^{-1} \mathrm{Mpc}^{-1}$ and $q_{0}=0$ is given in Col. 7 of Table 1 .

\subsection{The EUVE data}

The EUVE/Deep Survey photometer (DS) images were extracted from the raw event lists with the customary linear bin size of 13 pix/arcmin. The rectangular shape of the Lex/B filter $(\sim 70-200 \mathrm{eV})$ results in images where the length of one side of the image greatly exceeds the other side (see Fig. 1). When more than one observation was available for each target, images were coadded to improve the $S / N$; the rectangular shape often results in only partial overlap between the exposures leading to diamond-shaped geometries (see Fig. 2). Without taking into account any edge corrections, this peculiar shape of the EUVE images may be a problem for any analysis involving an isotropic distribution of the data at scales larger than about one-third the size of the shorter side.

Providing that the image does not truncate the cluster emission in any direction, this shape problem can be treated as a classical edge effect. A simple solution is then to use the asymptotic background values to obtain a simulated larger square image. From a practical point of view only a small truncation of the data occurs in our images along the shorter axis. Its impact on an azimuthal profile can therefore be considered as negligible and we thus decided to apply the above procedure to modify the geometry of the EUVE images. We now give details on the required background estimation.

Figure 1 shows the rebinned image of a long observation of the Virgo cluster. The cluster is clearly visible in the central area of the field of view. Three rectangles are superimposed on the raw image. The largest one, denoted by (A, B, C, D) follows

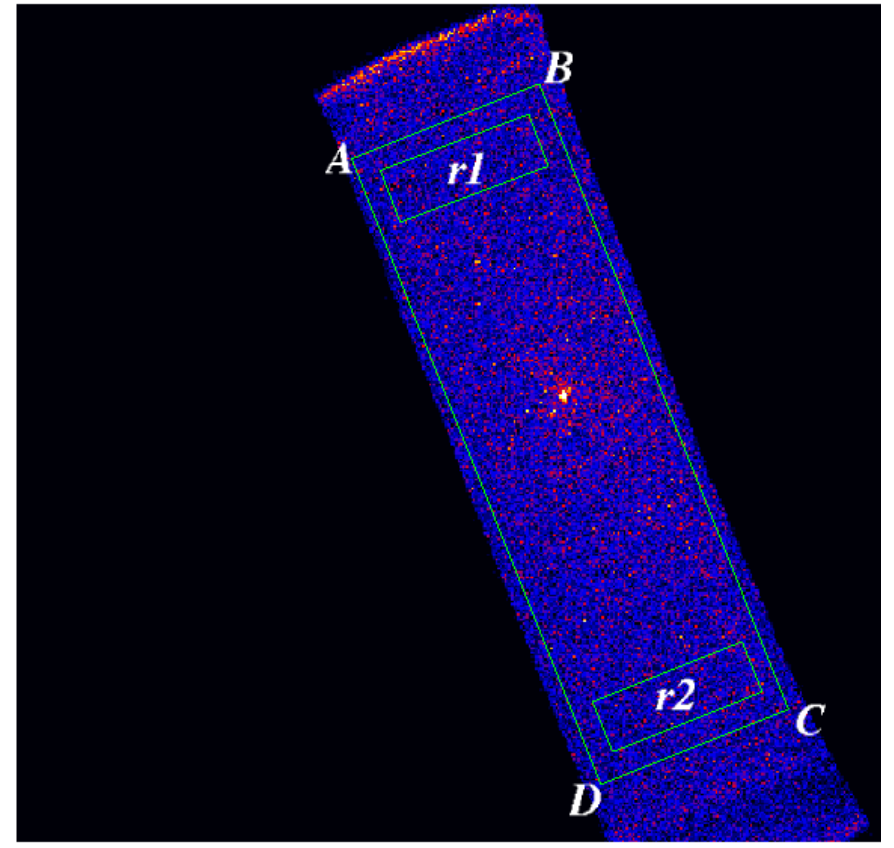

Fig. 1. Virgo long exposure - arrangement of boxes in order to obtain the image ready for the wavelet reconstruction. Note that the different boxes here are only illustrative of the algorithm we used.

the outer detector shape closely. Its edges are detached from the detector boundaries to avoid the steepening of the background near the edge, which would bias the noise estimation (see the Fig. 2 of Lieu et al. 1999c). In this box, we define two rectangles (denoted $r_{1}$ and $r_{2}$ ), one towards each edge, as far away from the central source as possible. Both $r_{1}$ and $r_{2}$ are assumed to contain only noise and to be representative of the noise level far from any celestial source. This is ensured because the centers of both rectangles lie at $\sim 40$ arcmin of the central source, a distance notably greater than the detection radius of the extended EUVE cluster emission, as seen with classical methods. The second order moment of the count distribution is used to estimate the level of the noise in each of these rectangles. The noise level in the large box is then computed as the mean of the noise levels in both rectangles. Once this has been done, each pixel outside the larger box is replaced by a random number drawn from a Poisson distribution, the variance of which is obtained by adding in quadrature the variances in each of the rectangles. The accuracy of the noise estimation is readily observable in the product image: if the noise is underestimated, the central box will obviously rise against the rest of the image, with its boundaries showing a clear discontinuity. On the other hand, if the noise has been overestimated, the outside parts of the new image will rise against the central box, again showing a discontinuity on the boundaries.

When more than one EUVE observation of a cluster was available, we used the co-added images in order to increase the signal to noise ratio. Figure 2 illustrates the case of Abell 1795. This image is typical of the co-added images we dealt with in the case of other clusters. The difference in roll-angle of the satellite in both observations is apparent. The central intersection of both observations obviously has the highest signal to noise ratio. It corresponds to a region indicated by the circle 
Table 1. Exposure times and main cluster characteristics.

\begin{tabular}{|c|c|c|c|c|c|c|c|}
\hline Cluster & $\begin{array}{l}\text { EUVE total } \\
\text { exp. time (s) }\end{array}$ & $\begin{array}{r}\text { ROSAT exp. } \\
\text { time (s) }\end{array}$ & $\begin{array}{c}\text { ROSAT } \\
\text { ObsId }\end{array}$ & Redshift & $\begin{array}{r}k T_{\mathrm{X}} \\
(\mathrm{keV})\end{array}$ & $\begin{array}{r}\text { Scaling } \\
(\mathrm{kpc} / \text { superpx })\end{array}$ & $\begin{array}{c}\text { Cooling } \\
\text { flow }\end{array}$ \\
\hline A 1795 & 158689 & 33921 & rp800105 & 0.063 & 5.9 & 32.7 & yes \\
\hline A 2199 & 93721 & 34633 & rp800644 & 0.0299 & 4.1 & 15.8 & yes \\
\hline A 4059 & 145389 & 5225 & rp800175 & 0.0478 & 4.0 & 25.0 & yes \\
\hline Coma & 60822 & 20112 & rp800005 & 0.023 & 8.7 & 12.2 & no \\
\hline Virgo & 146204 & 9135 & rp800187 & 0.003 & 2.4 & 1.6 & weak \\
\hline
\end{tabular}

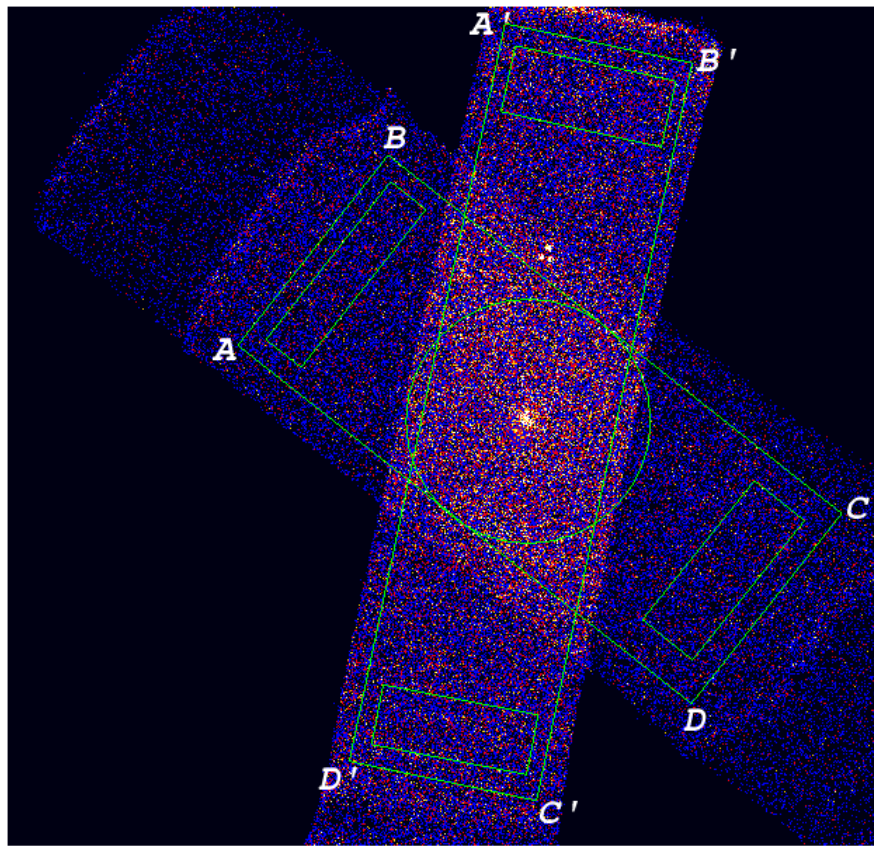

Fig. 2. Abell 1795 arrangement of boxes in order to obtain the image prior the wavelet reconstruction in the case of a co-added image. The central circle has a radius of $16 \mathrm{arcmin}$, which we will take as the limit of our analysis.

of radius 16 arcmin in Fig. 3, which we will take as the radial limit for our analysis. For each of the separate observations, we drew again a box closely following the outer detector shape. Both boxes are labelled $(\mathrm{A}, \mathrm{B}, \mathrm{C}, \mathrm{D})$ and $\left(\mathrm{A}^{\prime}, \mathrm{B}^{\prime}, \mathrm{C}^{\prime}, \mathrm{D}^{\prime}\right)$ in Fig. 2. In each of the boxes, we define two small rectangles, where the noise is evaluated. The noise level in a box is computed as the mean of the noise levels in both rectangles. Once this has been done for each box, each pixel outside the intersection of the boxes (the central diamond) is again replaced by a random number drawn from a Poisson distribution, as discussed before. Once again, the under or overestimation of the noise will be clearly visible. An example of such an image is shown in Fig. 3.

\subsection{The ROSAT data}

The ROSAT X-ray data for each cluster were obtained from the HEASARC web archive ${ }^{1}$. Owing to the higher sensitivity, we

\footnotetext{
${ }^{1}$ http://heasarc.gsfc.nasa.gov/
}

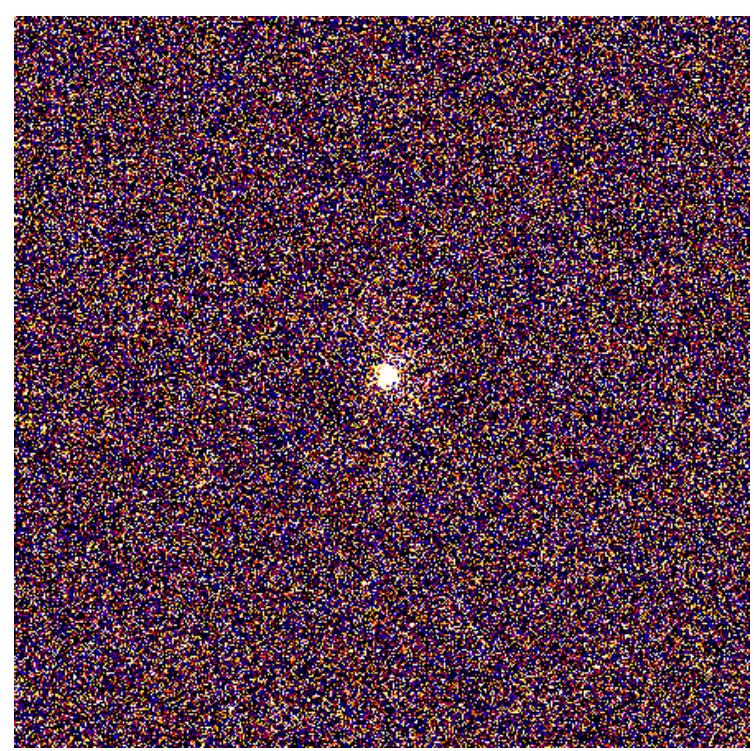

Fig. 3. Enlarged and rebinned image of Abell 1795. The total size of the image is $140 \times 140 \mathrm{arcmin}^{2}$.

preferred PSPC to HRI data and pointed observations to allsky survey scanning mode observations. When multiple PSPC observations were available, we always used the one with the smallest offset radius compared to the center of the cluster, and then the longest one.

S. Snowden's Extended Source Analysis Software (ESAS $\left.{ }^{2}\right)$ was used to perform the data reduction, i.e., rejection of highbackground times, modelling of the different background types, energy-dependent background subtraction, exposure and vignetting corrections (see Snowden et al. 1994). We adopted a conservative value of $170 \mathrm{cts} \mathrm{s}^{-1}$ for the maximum Average Master Veto rate allowed. We then carefully examined the light curves of the total counts in the entire image per energy band, and checked that no short time scale glitches were present. We limited our analysis to the $[0.5,2.0] \mathrm{keV}$ energy band (bands R4-R7 as defined by Snowden). This preliminary reduction produced a surface brightness image with $512 \times 512$ pixels, 15 arcsec per pixel (roughly the FWHM of the PSPC PSF at $1 \mathrm{keV}$ ), as well as an exposure image for each of the bands and a background image. The image was then divided by the mean exposure map. We thus obtained a $512 \times 512$ pixel image,

\footnotetext{
${ }^{2}$ ftp://legacy.gsfc.nasa.gov/rosat/software/fortran/ sxrb
} 
in units of counts $\mathrm{s}^{-1}$ pixel $^{-2}$, which was then rebinned to $18.5 \times 18.5 \operatorname{arcsec}^{2}$ pixels to match the EUV superpixel size.

The ROSAT images do not present the same problems as the EUVE ones, because in this case, the geometry is circular around the central cluster (all our observations were pointed). The only geometrical constraint arises from the presence at an offset angle of approximately 20 arcmin of the structure support of the telescope. However, since this value is smaller than the EUVE limiting radius, it does not place any further constraint here. We therefore just extracted a square image inscribed in the 20 arcmin radius circle.

\subsection{Wavelet analysis and reconstruction}

The comparison between the EUVE observed surface brightness profiles of clusters and their ROSAT X-ray predicted counterparts is the key step in our analysis. However a direct comparison of the X-ray and EUVE data would provide misleading results due to the noise in the raw data. To remove most of this noise while preserving information on the astronomical emission at all spatial scales of interest we decided to apply part of the wavelet vision model described in detail in Rué \& Bijaoui (1997).

Let us hereafter briefly summarize the main steps of the image processing involved. First, a discrete wavelet transform of the image $I_{0}$ is performed using the so-called à trous implementation, which is nothing more than the classical multiresolution algorithm of Mallat (1989) but without any decimations since "holes" are introduced in the required convolutions. The initial sampling is kept for each scale; this insures shiftinvariance and allows straightforward interscale computations. The scaling function leading through a dilation factor $a$ to the involved sets of low-pass and band-pass (wavelets) filters is a cubic B-spline (defined in the Fourier space as $\operatorname{sinc}^{4}(\omega)$ ), very well suited for enlightening Gaussian-like patterns of various sizes in an image. Owing to the properties of the underlying convolutions a stationary white noise signal leads to a statistical distribution of the wavelet coefficients with a zero mean value at each computed scale $a_{j}$ and a standard deviation decreasing as the spatial scale increases. Structures in the transform which are related to objects in direct space can then be identified and located by selecting high enough wavelet coefficients at each scale.

Removing noise from the data without any loss in resolution can thus be achieved quite easily in wavelet space by keeping only those coefficient values with a probability to be due to a chance fluctuation of the noise process lower than a chosen decision level. This second step of the noise removal process requires the knowledge of the probability density function of the coefficients at scale $a_{1}=1$ pixel (here $18.5 \operatorname{arcsec}$ ) for the noise process and of the decreasing law of the rms scatter $\sigma$, the asymptotic value of which appears to be 0.5 for a bidimensional image $I_{0}$ and a dyadic scale analysis $\left(a_{j}=2^{j-1}, j \in \mathcal{Z}\right)$ : $\sigma_{j}=\sigma_{1} / 2^{j-1}$. In case of Gaussian noise, the probability density function is easily obtained from a histogram of the wavelet coefficients providing that this first scale of one pixel in size is noise dominated. Due to the stationarity of the Gaussian process, the selection of the so-called statistically significant coefficients $v_{j}$ at scale $a_{j}$ can then be done using the same $k$ $\sigma_{j}$ threshold. We end up in this way with a subset $v^{(0)}$ of coefficients at different scales, the pixels of which define what is called hereafter the multiscale significant mask $M_{0}$. In case of Poisson noise stationarity can be obtained by applying an Anscombe (1948) transform on the data: $f(x)=2 \sqrt{x+0.375}$. When the mean parameter $\mu$ of the Poisson process is greater than about 5, this transform indeed leads to a new process with a very small bias in the expectation value $\left(\frac{<f(x)>-f(\mu)}{f(\mu)}<2 \%\right)$ and a nearly perfect variance stabilization. The same decision scheme as in the Gaussian case can therefore be used for defining the multiscale significant mask from the wavelet transform of this image with a constant variance. Only second order errors in the wavelet coefficients would arise at the very first scales in areas with very low counts. This is not a matter of concern for the present application since the computation of a correct azimuthal brightness profile necessitates only a proper account of the medium and large-scale features in these faint regions.

The third and final step is to restore a positive image $I$ where the noise is strongly reduced from the subset $v^{(0)}$ of the significant wavelet coefficients of $I^{(0)} \equiv I_{0}$. This is an inverse problem which has in general an infinity of solutions and the choice among these solutions must be done with respect to a regularization constraint. To do so, an iterative conjugate gradient algorithm is used. This algorithm minimizes the distance $\left\|v^{(0)}-v^{(n)}\right\|$ where $v^{(n)}$ is the restriction to $M_{0}$ of the wavelet transform of the solution $I^{(n)}$ at step $n$. Stating $A$ the operator associated to the wavelet transform followed by the projection onto $M_{0}$ and $\tilde{A}$ the joint operator, $I^{(n+1)}=\tilde{A}\left(v^{(n)}\right)$ is obtained from the previous solution by: $I^{(n+1)}=I^{(n)}+\alpha^{(n)} I_{\mathrm{r}}^{(n)}$ where the residual image $I_{\mathrm{r}}^{(n)}$ is defined as $\tilde{A}\left(v_{\mathrm{r}}^{(n)}\right)+\beta^{(n)} I_{\mathrm{r}}^{(n-1)}$ with $v_{\mathrm{r}}^{(n)}=v^{(0)}-\mathrm{A}\left(I^{(n)}\right) ; \alpha$ and $\beta$ are convergence parameters. Convergence $\left(\frac{\left\|v^{(0)}-v^{(n)}\right\|}{\left\|v^{(0)}\right\|}<1 \%\right)$ is reached after very few steps, leading to an image $I$ without any discontinuities despite the hard thresholding performed in the wavelet space. Note also that any flat instrumental or astronomical component or gradient in the initial image is automatically removed in image $I$ taking benefit of the mathematical properties of the wavelet function related to our scaling function.

We applied such an image processing to both our EUVE and ROSAT square images considering seven or eight spatial scales for the wavelet transform according to the size of the image. Pure Poisson noise was considered in both cases. This is not strictly the case for the ROSAT data because of the preprocessing of the raw counts (exposure correction, modelled background subtraction), but this approximation appears to be good enough for detecting correctly all the large-scale components we are interested in. Significant wavelet coefficients were thus selected according to a probability of false detection of $4 \sigma, 3.5 \sigma$ and $3 \sigma$ for scales 1 (18.5 arcsec), 2 (37 arcsec) and 3 (74 arcsec) and above respectively. The rather high levels for the first two scales automatically eliminate most of the smallscale structures if any (these might distort the overall shape of the brightness profile or might have arisen from an error in the probability density function of the noise or from a loss in control over the bias in the Anscombe transform in regions with 
very few counts). We have checked in several cases that the profiles do not change significantly when the thresholding for scales 1,2 and 3 is modified.

\subsection{Radial profiles}

After wavelet reconstructing both EUVE and ROSAT images for each cluster, we proceed to compare the decrease of the surface brightness in the two observational bands. Profiles were thus derived from the $2 \mathrm{D}$ reconstructed images within concentric elliptical rings using the STSDAS.ANALYSIS. ISOPHOTE.ELLIPSE task in IRAF. The cluster ellipticities and major axis position angles were estimated from the X-ray images, which have better signal to noise ratio, except for Coma because it fills the 20 arcmin PSPC ring and its ellipticity had to be derived from the EUVE reconstructed image. The ellipticities and major axis position angles were then fixed to be the same in the EUV and X-rays, in order to measure intensities in the same spatial regions.

In order to see how the instrument point spread functions (PSF) could influence our results, we also analyzed a point source in a similar way: the star $\gamma$ Tau, for which both EUV and X-ray data were available. The EUV and X-ray exposure times for this star were $89924 \mathrm{~s}$ and $8547 \mathrm{~s}$ respectively, and the ROSAT PSPC image of $\gamma$ Tau was divided by its corresponding exposure map, as for clusters. The EUV and X-ray images were rebinned to a pizel size of $18.5 \times 18.5 \mathrm{arcsec}^{2}$ as previously, and the EUV and X-ray profiles were then derived in a similar way. Note that this point source is about 15 arcmin offset from the detector center in the ROSAT image, at a radius where the PSF of PSPC is already degraded. Therefore, the PSF effect in X-rays will in fact always be smaller or equal than what we measure for $\gamma$ Tau. This is not the case for EUVE, where the point source is close to the detector center.

The problem of a possible non-flatness of the background was already addressed in the case of EUVE by Lieu et al. (1999c). A comparable wavelet analysis was performed on a blank field totalling $85 \mathrm{ksec}$ exposure time, and the backgroud was found to vary by at most $10 \%$ within the central circle of 16 arcmin radius defined above taken into account here (see Fig. 1 by Lieu et al. 1999c). The mean value of another blank field observed for $21 \mathrm{ksec}$ is found to be 1.21 counts $/ \mathrm{s} / \mathrm{pixel}^{2}$, excluding the outermost regions (that is in a rectangle similar to ABCD from Fig. 1), which is comparable to the mean background value in Abell 1795 (1.19 counts/s/pixel $\left.{ }^{2}\right)$.

In the case of the X-ray images, they were divided by their corresponding exposure maps, and therefore the non-flatness of the ROSAT PSPC detector should be corrected for. We are therefore confident that the non-flatness of the detector backgrounds cannot change our results by more than a few $\%$.

Error bars on all profiles are those directly given by the ELLIPSE task. However, since we were concerned that they might be underestimated, we also computed them from the Poisson fluctuation in the number of counts in each ring; these error bars were found to be commensurate with those given by ELLIPSE (the rms fluctuation of the pixel intensity within each concentric ellipse). For reconstructed images, they are typically

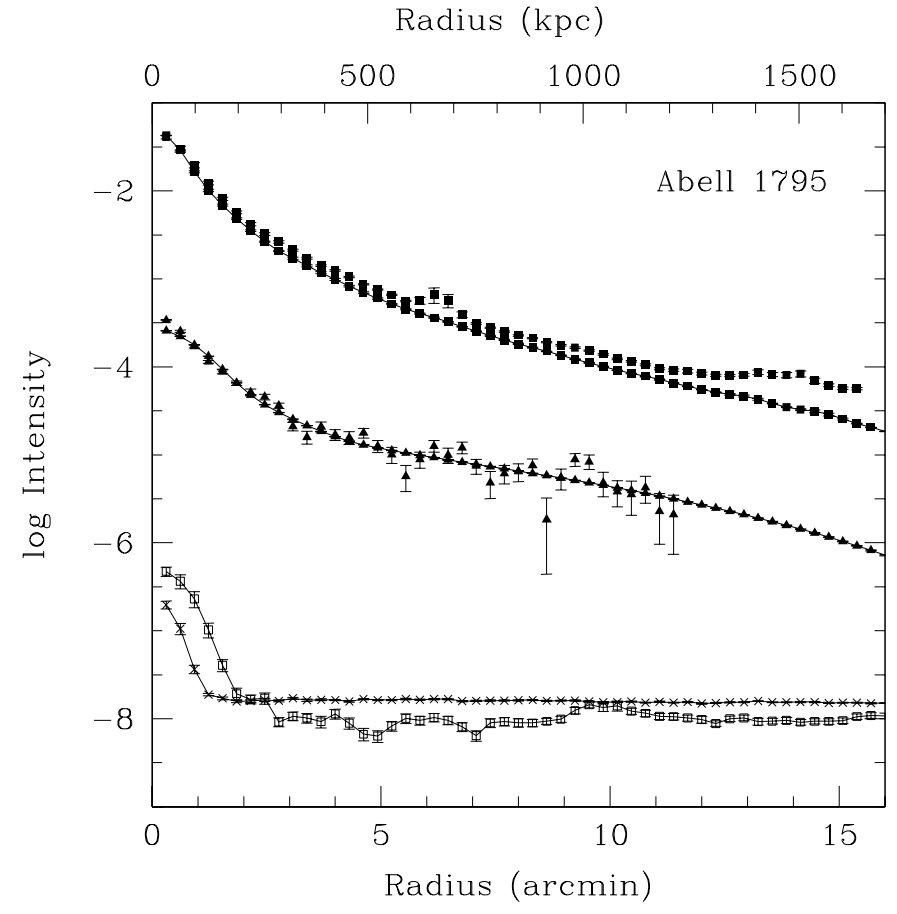

Fig. 4. EUV and X-ray profiles of Abell 1795, obtained directly from the raw data (points) and after wavelet analysis and reconstruction (points with connecting lines). As in the four following figures, the EUVE data are drawn with triangles (bottom curves) and the X-ray data with filled squares (top curves); the radius is expressed in arcminutes (bottom) and kpc (top); intensities are in counts $/ \mathrm{s} / \mathrm{pixel}^{2}$ after background subtraction ( 1 pixel is $18.5 \times 18.5 \operatorname{arcsec}^{2}$ ). The error bars are drawn, but for the profiles derived from the wavelet reconstructed images they are too small to be clearly visible (typically smaller than \pm 0.01 in logarithmic scale for Figs. 4 to 8 ). The EUV (crosses) and ROSAT PSPC (empty squares) PSFs drawn from the images of the point source $\gamma \mathrm{Tau}$, shifted by an arbitrary value to make the figure clearer, are shown at the bottom for comparison.

smaller than \pm 0.01 in logarithmic scale, i.e. too small to be clearly visible on Figs. 4 to 8).

\section{Results}

\subsection{EUV and X-ray profiles}

For co-added images, we do not expect to detect any structure outside a certain radius since the outer part of the image is purely noise. The central circle in Fig. 2 shows a conservative estimate of this radius, i.e. the half width of the EUVE detector $\sim 16$ arcmin, which we will take as the limit of our analysis.

We first derived the profiles of the EUVE and X-ray images of all clusters before and after wavelet analysis and reconstruction, as a test to check that our method conserves the overall profile shape and intensity level. The corresponding profiles are shown in Fig. 4 for Abell 1795. Note that all the profiles drawn in Figs. 4 to 8 are obtained after subtracting the background. For the raw data, this background is estimated as a mean value far from the edges of the detector; for wavelet reconstructed images, the background is modelled during the wavelet analysis itself and subtracted. There is good consistency between the two EUVE curves on the one side and between the two X-ray 


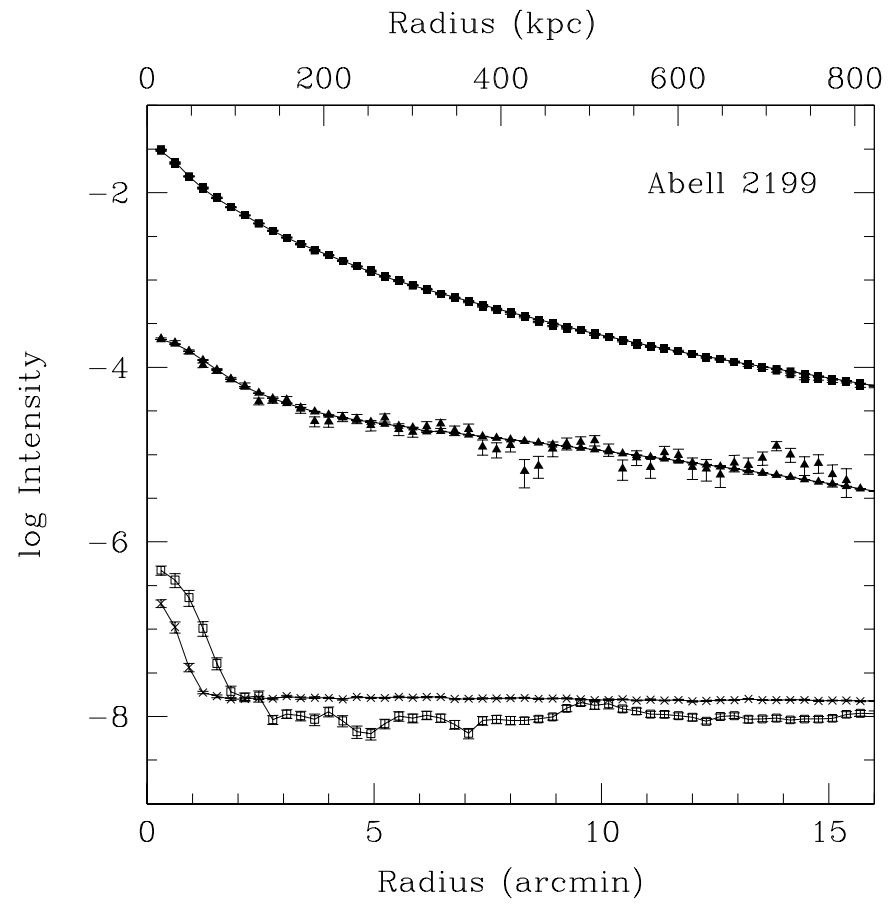

Fig. 5. Same as Fig. 4 for Abell 2199.

curves on the other side, implying that the wavelet analysis and reconstruction applied here does not affect fluxes, but simply provides a means of detecting cluster emission out to a somewhat larger radius (as in the case of the EUVE data). The small feature apparent in the X-ray profile at $\sim 6$ arcmin is due to a Seyfert galaxy superimposed on the cluster; it was removed from the wavelet reconstructed image and therefore does not appear on the corresponding profile. Note however that Abell 1795 is the only cluster for which the raw and wavelet reconstructed X-ray profiles do not coincide exactly (in the other clusters they can hardly be distinguished). Although we have tried various thresholding values for the small scales and various background subtractions of the raw data to see if they could coincide better, we have not been able to superimpose them exactly.

The EUV and ROSAT PSPC PSFs drawn from the images of a point source (see Sect. 2.5.) for comparison are also plotted in Fig. 4. They indicate that the PSFs have no influence on the profile shapes for radii larger than about 1 arcmin and 2.5 arcmin in the EUV and X-rays respectively. Therefore any features found in the profiles within regions smaller than these radii are probably not real.

The EUV and X-ray radii of Abell 1795 are at least 16 arcmin, corresponding to a physical extent of about $1700 \mathrm{kpc}$. This extent is notably larger than the previously found value of $10 \mathrm{arcmin}$ (Mittaz et al. 1998; Bonamente et al. 2001b).

The EUV and X-ray radii of Abell 2199 extend to at least 16 arcmin, or $820 \mathrm{kpc}$ (Fig. 5), comparable to the value of 15 arcmin measured by Kaastra et al. (1999) both with EUVE and Beppo-Sax.

In Abell 4059 (Fig. 6), the EUV and X-ray emissions also reach 16 arcmin or $1300 \mathrm{kpc}$, much further than the 4-5 arcmin

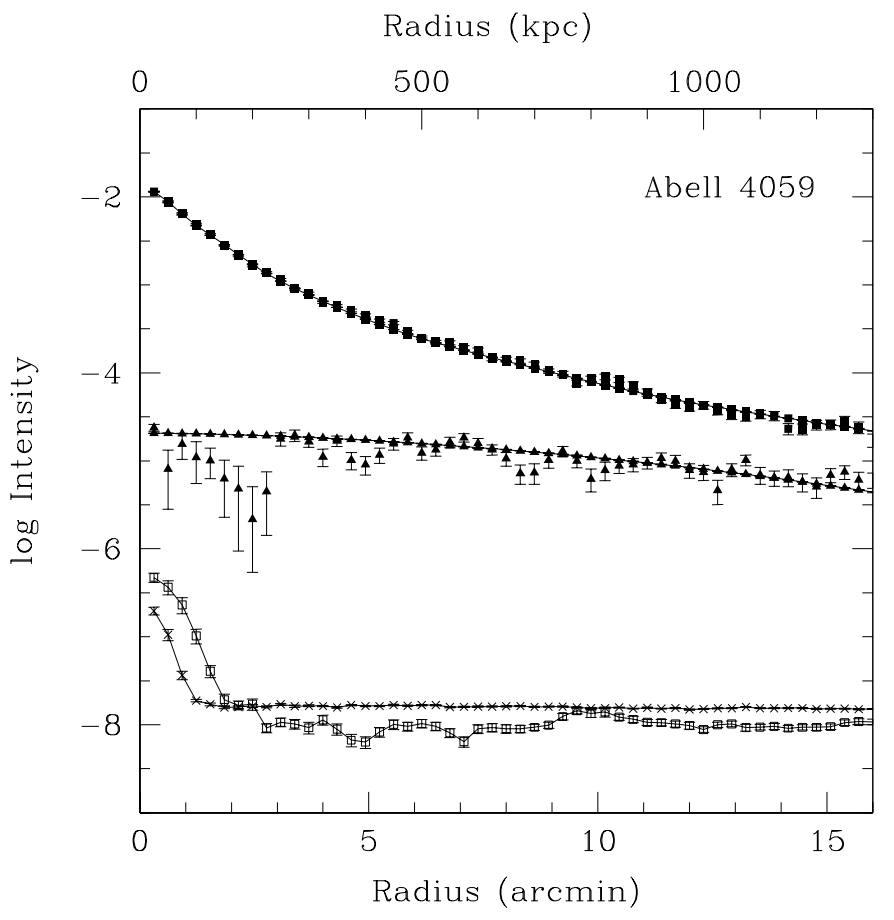

Fig. 6. Same as Fig. 4 for Abell 4059.

reported by Berghöfer et al. (2000b). The slopes of the profiles in the EUV and X-rays differ notably, at least in the inner 8 or 10 arcmin. The difference in the central regions between the EUV profiles derived from the raw and wavelet reconstructed images may be due to the fact that structures only present at small scales were eliminated, and therefore signal may be missing in the very center. This cannot be due to the shape of the EUVE PSF, since it is flat for radii larger than about 1 arcmin while this discrepancy is observed up to a radius of about 3 arcmin. Such a behaviour is not observed in Abell 1795, Abell 2199 or Coma, probably because they are brighter.

The EUV and X-ray radii in Coma reach at least 16 arcmin (Fig. 7), corresponding to $630 \mathrm{kpc}$ and slightly larger than reported by Bowyer et al. (1999). The two profiles appear quite similar at least up to a radius of about $10 \mathrm{arcmin}$.

Virgo is the most nearby cluster in our sample. EUV and $\mathrm{X}$-ray emissions reach at least 16 arcmin (Fig. 8), that is slightly larger than detected by Lieu et al. (1996a) and Berghöfer et al. (2000a), but smaller than the 20 arcmin claimed by Bonamente et al. (2001b); however, this extent corresponds to the very small physical value of $\sim 80 \mathrm{kpc}$, due to the proximity of Virgo. Both profiles appear quite parallel except in the inner 4 arcmin; note however that since the X-ray PSF is not flat for radii smaller than 2.5 arcmin, the difference between the EUV and $\mathrm{X}$-ray profiles in the inner 4 arcmin may not be real.

In order to quantify the probability for the EUV and X-ray profiles to be similar, we performed a Kolmogorov-Smirnov (hereafter K-S) test on the EUV and X-ray profiles drawn from the wavelet reconstructed images, both in concentric elliptical annuli, and in ellipses with increasing radius, after normalizing the EUV and X-ray profiles to the same innermost pixel value. 


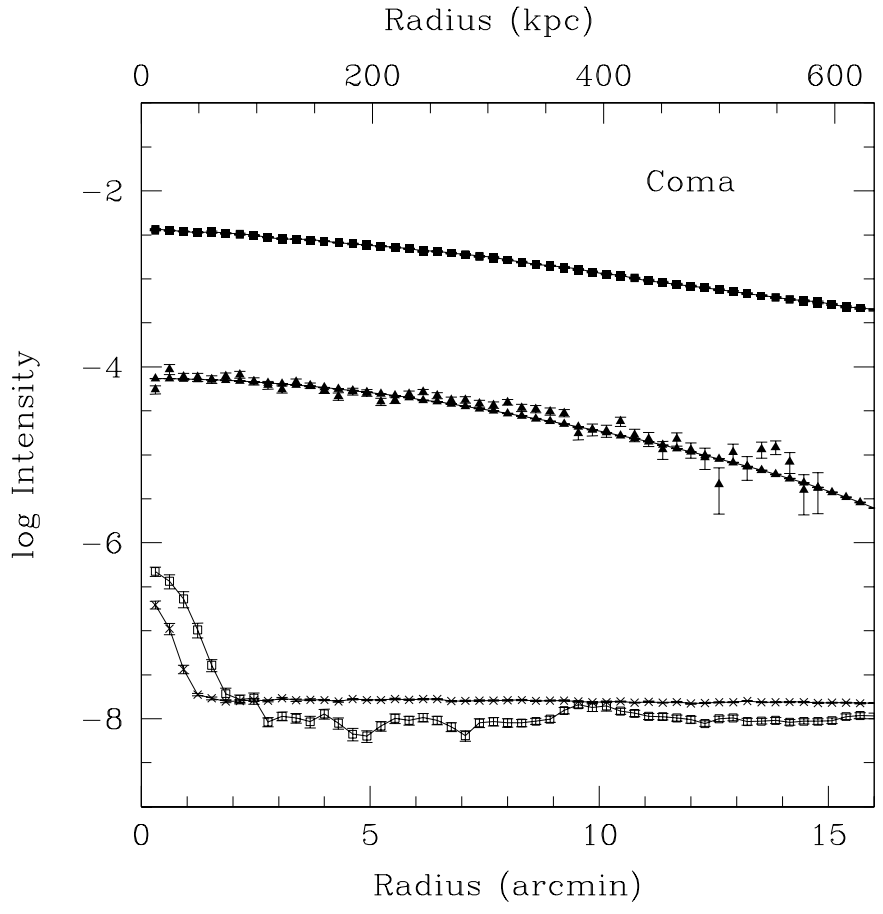

Fig. 7. Same as Fig. 4 for Coma.

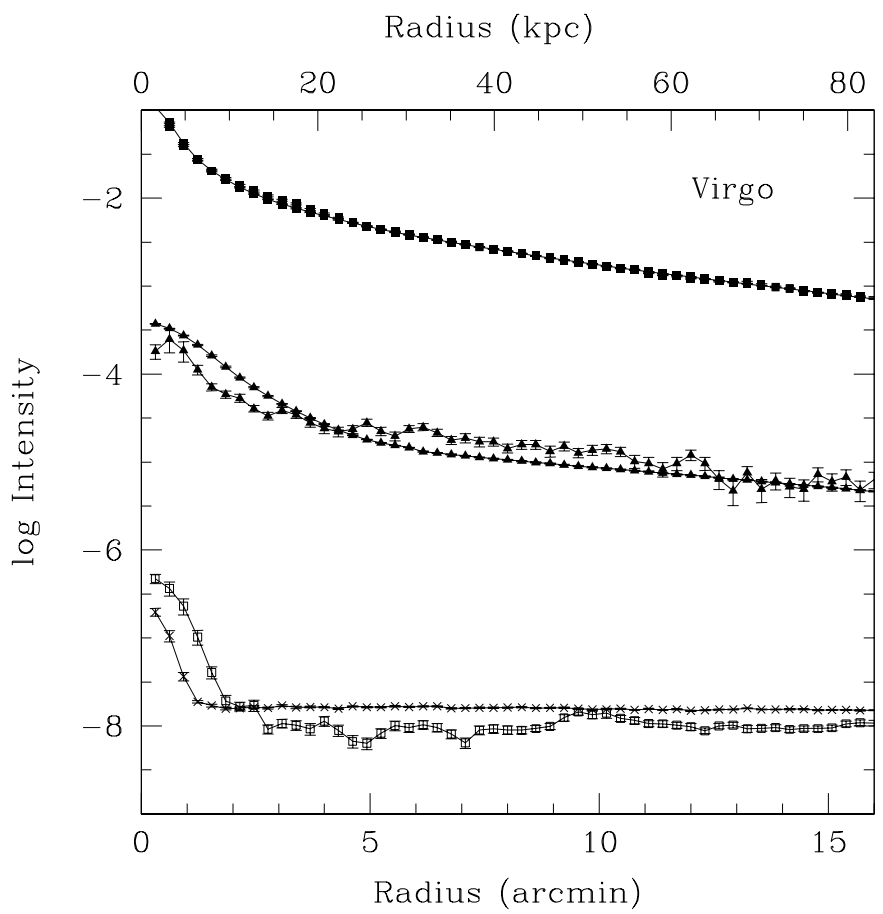

Fig. 8. Same as Fig. 4 for Virgo.

In all clusters, the probability that the two profiles are issued from the same parent population varies with radius: it is a few $\%$ in the very center ( 1.5 arcmin radius), and less than $2 \%$ in concentric annuli of width 1.5 arcmin (i.e. for radii between 1.5 and 3.0, 3.0 and 4.5 arcmin etc.). If we consider ellipses of radii 3.0, 4.5, 6.0 arcmin etc. instead, the probability that the two profiles are issued from the same parent population is smaller than $0.1 \%$ in all cases. Therefore even if the EUV and
$\mathrm{X}$-ray intensity profiles may appear similar to the eye, as for example in Coma, it is clear that statistically they are not.

\subsection{EUV to $X$-ray intensity ratios}

In order to compare the EUV to X-ray profiles, we computed the EUV to X-ray intensity ratios in the same concentric ellipses; these are shown in Figs. 9 to 13. The ratio of the EUV to X-ray PSFs was also derived (see Fig. 9). As expected from the individual EUV and X-ray PSFs, this ratio is only constant for radii larger than 2.5 arcmin, while below it shows a strong dip. Therefore the EUV to X-ray ratios below this radius are not reliable. Such ratios have to be compared to those expected in the hypothesis that the EUV emission is the low energy tail of the hot ICM emission. We therefore employed spatially resolved hot ICM models for each cluster, in order to account for the effects of temperature and abundance gradients on the predicted ratios. Spectra of all cluster regions were modelled with a thin-plasma emission code (MEKAL in XSPEC, Mewe et al. 1985, 1986; Kaastra 1992) modified by photoelectric absorption (WABS in XSPEC, Morrison \& McCammon 1983). In detail, the hot ICM in Abell 1795 was modelled with $k T$ increasing from $3.5 \mathrm{keV}$ at the center to $6 \mathrm{keV}$ at radii $\geq 3$ arcmin, and abundances in the 0.3-0.5 solar range (Briel \& Henry 1996); Abell 2199 was modelled with $k T$ in the $2.5-4 \mathrm{keV}$ range, and abundances decreasing from $\sim 0.7$ solar at the center to 0.3 solar for radii $\geq 3$ arcmin (Siddiqui et al. 1998); Abell 4059 was modelled with $k T$ increasing from $2 \mathrm{keV}$ at the center to $5 \mathrm{keV}$ at radii $\geq 3$ arcmin, and abundances in the 0.3-0.6 solar range (Hwang \& Sarazin 1998); Coma was considered as isothermal with $k T=8.2 \mathrm{keV}$ and abundances 0.21 solar (Hughes et al. 1993); finally, the hot ICM parameters of Virgo were directly fit to our PSPC data, with $k T$ in the $1.2-3 \mathrm{keV}$ range, and metal abundances of $0.4-0.6$ solar. Using these models, we were able to derive the expected count rates in the EUV DS band and in the PSPC $1 / 4 \mathrm{keV}$ band, taking into account the effective area of the instruments and the performances at all detector positions. The points corresponding to these calculated count ratios are shown in the following figures.

In Abell 1795, the EUV to X-ray intensity ratio (Fig. 9) is smaller than 1 in the center (for radii smaller than 1.5 arcmin), but this is most probably due to the shape of the ratio of the EUV to X-ray PSFs. It then increases with radius to reach a roughly constant value of 4 . The shape of this curve is consistent with that given by Bonamente et al. (2001b), while the value of 4 that we find in the outer regions is intermediate between that of 6 given by Mittaz et al. (1998) and that of 2 given by Bonamente et al. (2001b). Error bars are typically smaller than \pm 0.01 in logarithmic scale, and are therefore difficult to see on the graphs. We therefore confirm the strong soft excess found in this cluster by these two groups of authors, and disagree with Bowyer et al. (1999) who claimed that no excess EUV emission was seen in this cluster. Note that the small disagreement between the raw and wavelet reconstructed X-ray image profiles (Fig. 4) cannot be at the origin of the EUV excess that we observe, since on the contrary the raw X-ray flux 


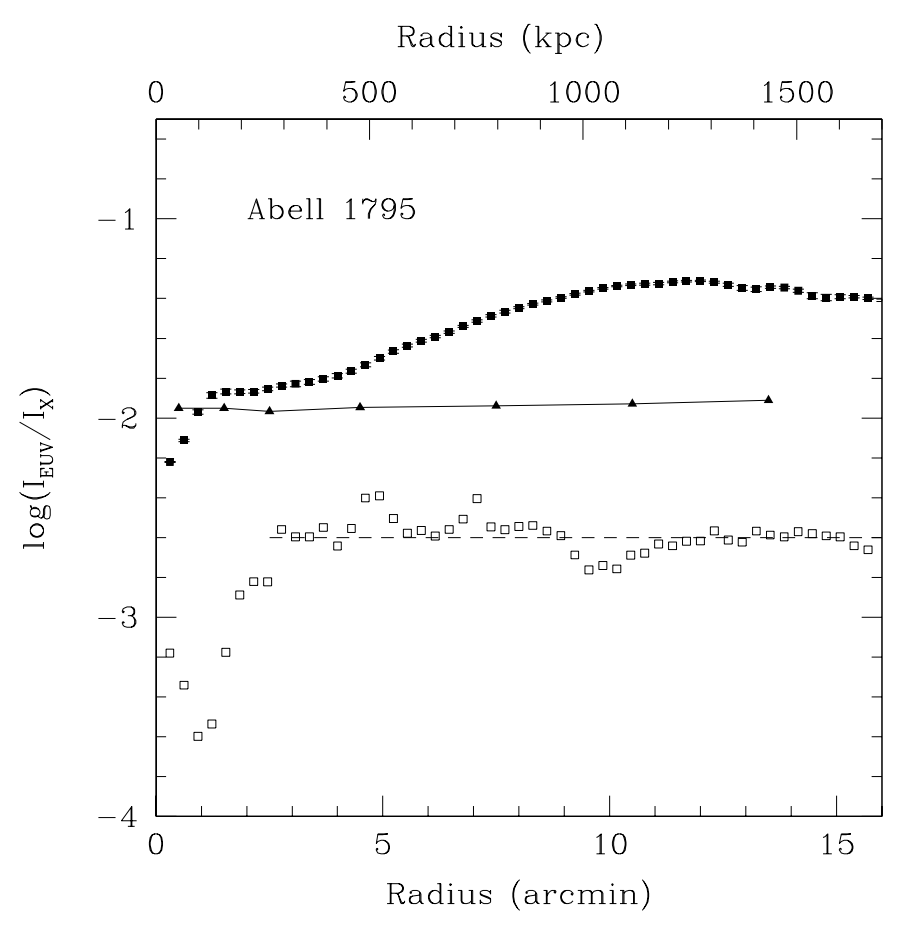

Fig. 9. Ratio of the EUV to X-ray emission in Abell 1795 in logarithmic scale. As in all following figures, squares show the measured EUV to X-ray flux ratios (error bars are difficult to see on the graphs since they are typically smaller than \pm 0.01 in logarithmic scale, as for the other clusters in Figs. 10-13). Triangles indicate the EUV to X-ray flux ratios in various concentric rings, expected from the hypothesis that the EUV emission is the tail of the bremsstrahlung emission accounting for the observed X-rays (see text). The ratio of the EUV to $\mathrm{X}$-ray PSFs is also drawn as empty squares, shifted by an arbitrary value to make the figure clearer; to guide the eye, the mean value of this ratio is indicated with a dashed line.

is higher that the reconstructed one, and therefore using the raw $\mathrm{X}$-ray data would lead to an even larger EUV excess.

The EUV to X-ray intensity ratio in Abell 2199 is smaller than 1 for radii below 5 arcmin, but as for Abell 1795 this is most probably due to the shape of the ratio of the EUV to X-ray PSFs. It then increases and reaches a value of about 3.7 between radii of 10 and 16 arcmin. We therefore agree with the soft excess observed in this cluster from various sets of data by Kaastra et al. (1999), and here also disagree with Bowyer et al. (1999) who found no excess EUV emission in this cluster.

The EUV to X-ray intensity ratio in Abell 4059 is smaller than 1 for radii below 2 arcmin (Fig. 11), in agreement with Berghöfer et al. (2000b), but again this is most probably due to the shape of the ratio of the EUV to X-ray PSFs; for increasing radii, it strongly increases and reaches a value of almost 20 between radii of 12 and 16 arcmin, in total disagreement with Berghöfer et al. (2000b), who find no EUV emission beyond 4 arcmin and no EUV excess beyond 2 arcmin.

Contrary to what happens in the three previously studied clusters, the EUV to X-ray intensity ratio in Coma appears rather flat up to a radius of at least 10 arcmin (Fig. 12), but as shown above the two distributions are not similar. The fact that no dip is seen within the central few arcminutes, as would be expected from the shape of the ratio of the EUV to X-ray

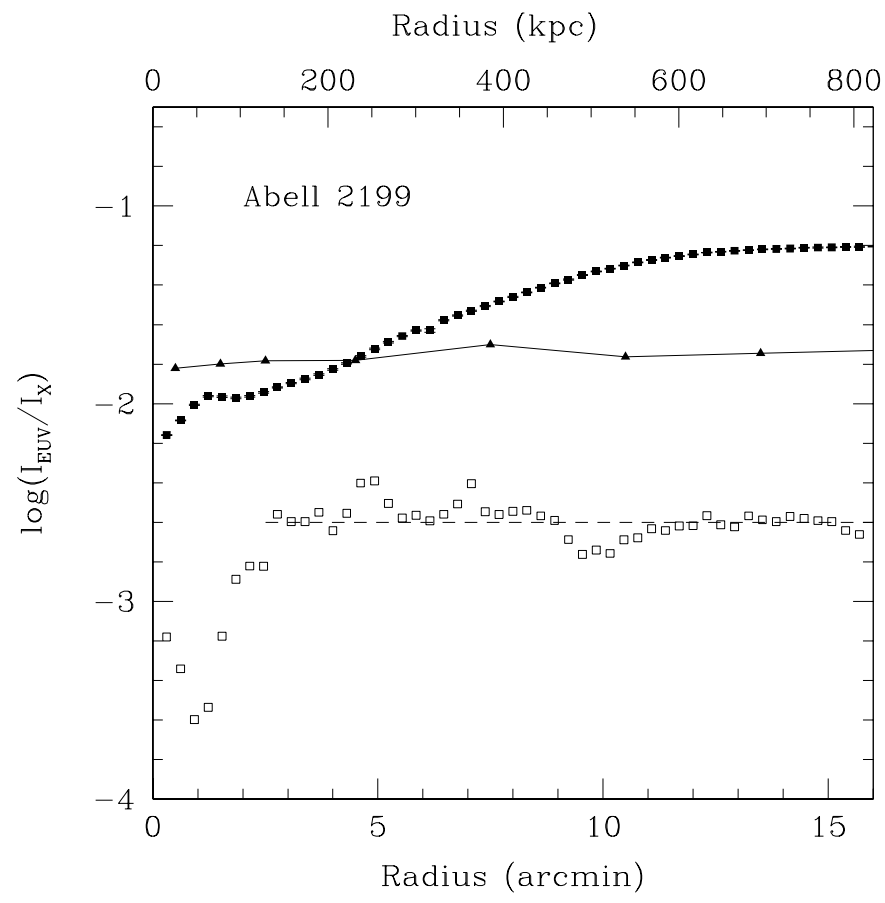

Fig. 10. Same as Fig. 9 for Abell 2199.

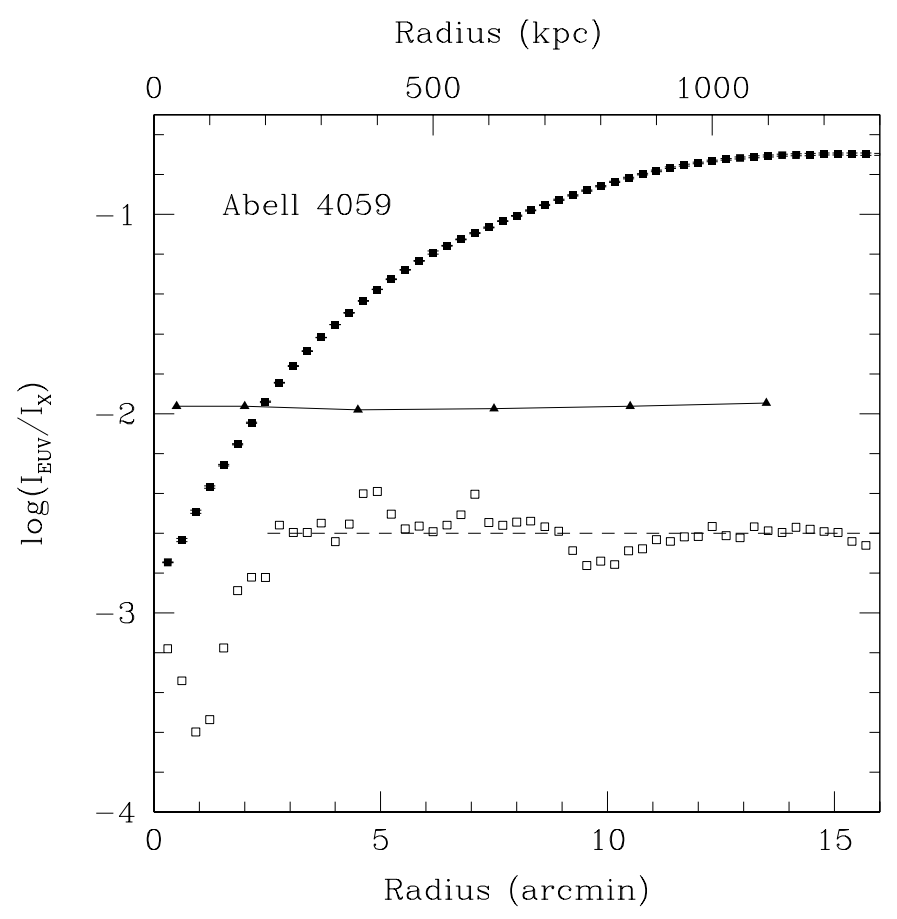

Fig. 11. Same as Fig. 9 for Abell 4059.

PSFs could indicate that the EUV excess in the center is even stronger than seen in Fig. 12. There is an EUV excess by a factor of $\sim 1.4$ over a thermal bresmsstrahlung tail.

The EUV to X-ray emission ratio in Virgo is in agreement with the EUV excesses previously reported by Berghöfer et al. (2000a) and Bonamente et al. (2001b), therefore implying that here also the EUV emission is not a thermal bremsstrahlung tail. The rise and fall of the EUV to X-ray ratio for radii increasing between 0.3 and 2.5 arcmin (Fig. 13) is rather surprising 
Radius (kpc)

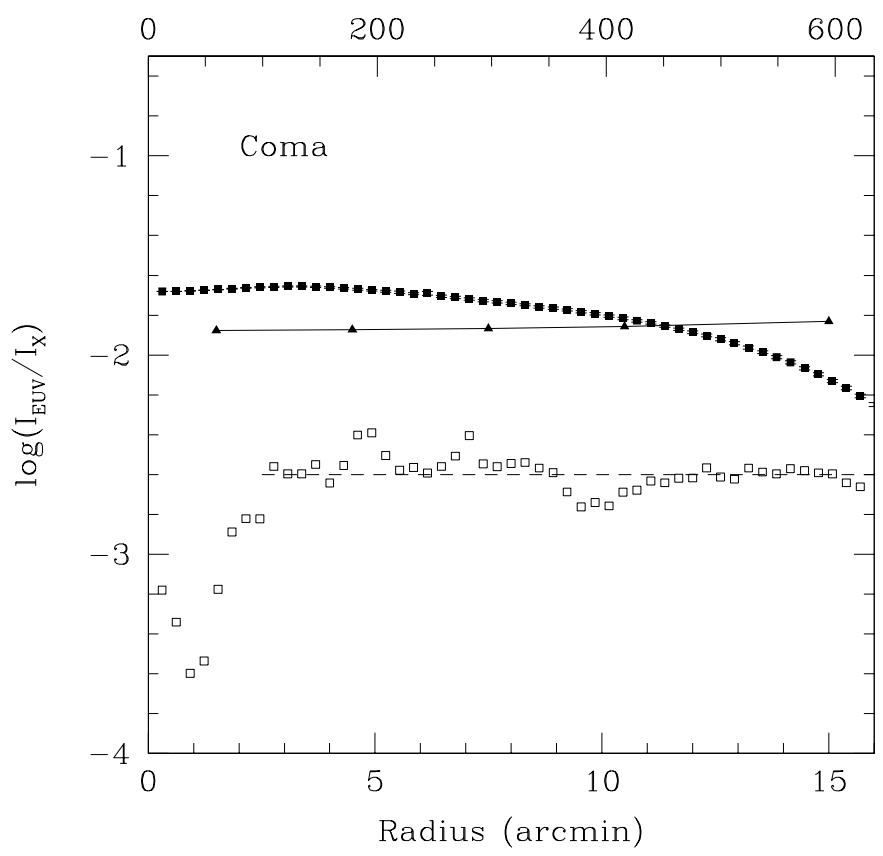

Fig. 12. Same as Fig. 9 for Coma.

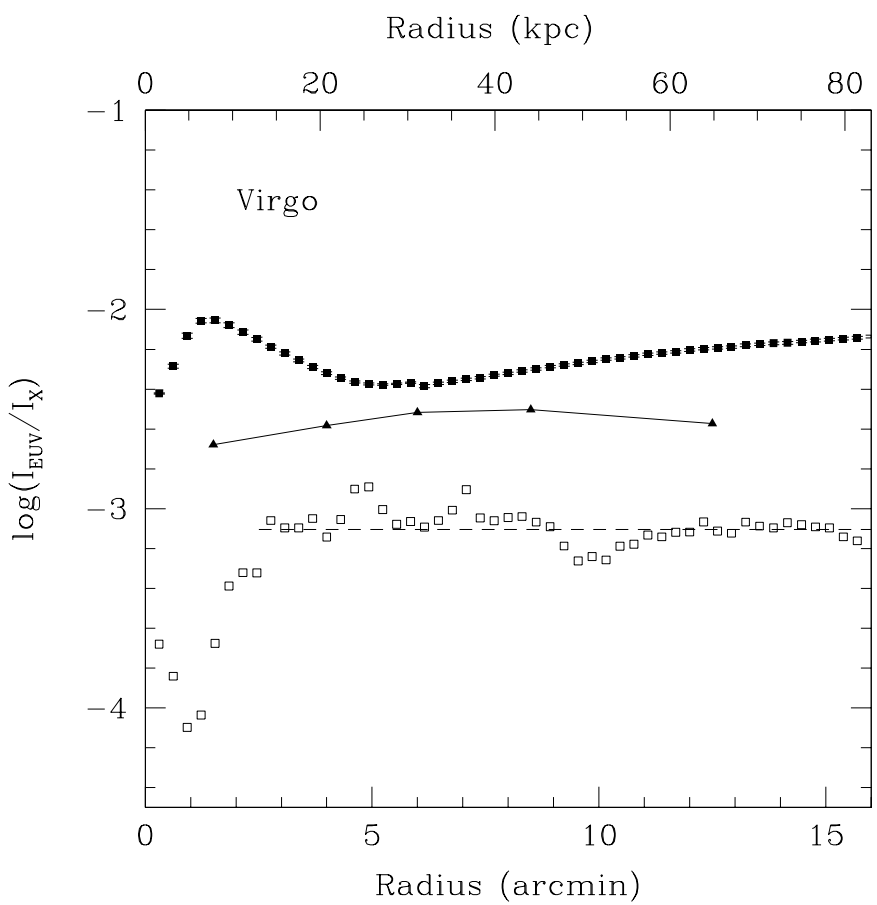

Fig. 13. Same as Fig. 9 for Virgo.

since it varies in an opposite way to the ratio of the EUV to $\mathrm{X}$-ray PSFs; however images with higher spatial resolution are obviously necessary to push the analysis further at these radii.

The superimposed EUV to X-ray intensity ratios for all the clusters of our sample are plotted in Figs. 14 and 15 for radii expressed in arcminutes and in kpc respectively. Although the shapes of these curves in the central 2.5 arcmin most probably reflect that of the ratio of the EUV to X-ray PSFs (Fig. 14), we can see that at least beyond this radius all the clusters do not
All clusters

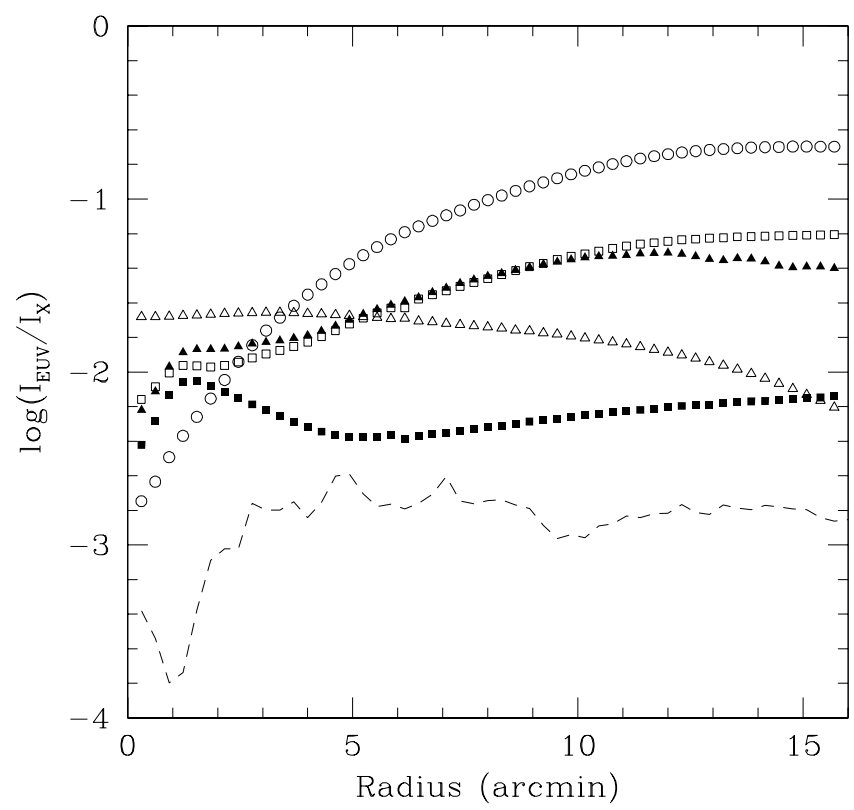

Fig. 14. EUV and X-ray profiles for the five clusters in our sample, with the radius expressed in arcminutes. The symbols are the following: Abell 1795: filled triangles, Abell 2199: empty squares, Abell 4059: empty circles, Coma: empty triangles, Virgo: filled squares. Error bars are omitted for clarity. The dashed line shows the ratio of the EUV to X-ray PSFs.

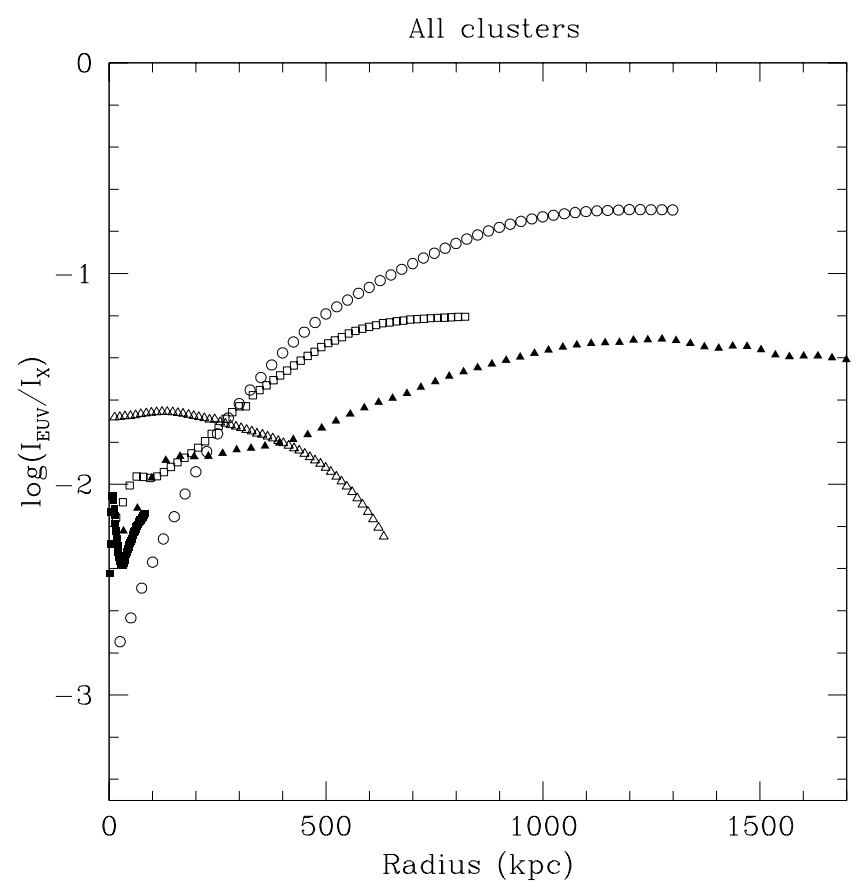

Fig. 15. EUV and X-ray profiles for the five clusters in our sample, with the radius expressed in kpc.

have the same behaviour. This is particularly true when radii are expressed in $\mathrm{kpc}$, and certainly must be linked to a difference in the emission mechanisms of the EUV emission in these clusters. Note that the radial extent of the EUV and X-ray emission in Virgo is comparable to the other clusters when the 
radius is expressed in arcminutes, but becomes extremely small in physical distance units, due to the very small redshift of the cluster: slightly more than $80 \mathrm{kpc}$.

\section{Discussion and conclusions}

In this paper, we have shown unambigously the existence of a EUV excess in all five clusters of our sample.

In the first three clusters (Abell 1795, Abell 2199 and Abell 4059), the EUV to X-ray intensity ratios have comparable behaviours: they show a possible deficiency of EUV emission over a bremsstrahlung tail in the very central regions (but this may be an effect of the ratio of the EUV to X-ray PSFs), and a EUV excess beyond a few arcmin. As suggested by several authors (Bowyer et al. 1999; Lieu et al. 2000), the first of these features, if real, can be interpreted as due to excess absorption within the cluster core due to the fact that in the cooler central regions some metals are not fully ionized and these ions absorb part of the soft X-ray flux. The presence of such excess absorption would agree with the previously claimed existence of cooling flows in these three clusters. However, XMM-Newton has detected much weaker emission lines than expected from bona fide isobaric cooling flow models, implying that there is significantly less "cool" gas than predicted (e.g. Kaastra et al. 2001). Therefore it is no longer straightforward to interpret excess absorption at the center of these clusters as due to the presence of cooler gas.

One may however note that the only cluster (Coma) for which no EUV dip is seen in the very center is both the hottest one by far and the only one with no "cooling flow" whatsoever. Since the ratio of the EUV to X-ray PSFs shows a dip for radii smaller than about 2.5 arcmin, the absence of such a dip in Coma suggests that in fact there is a significant EUV excess in the central regions of Coma.

Virgo shows a different behaviour, since the EUV excess is the strongest in the very center, within radii smaller than about 4 arcmin (corresponding to a physical size of $\sim 20-30 \mathrm{kpc}$, that is roughly the size of $M$ 87), then remains roughly constant with radius between 4 and 7 arcmin, and finally increases with a shallow slope for radii larger than 7-8 arcmin. As for Coma, the presence of a bump in the central regions suggests that the EUV excess is in fact very strong in this zone. For these last two clusters, images with higher spatial resolution are obviously necessary to analyze the EUV excess at these small radii.

The mere presence of an EUV excess in all five clusters of our sample indicates that a mechanism other than bremsstrahlung of the hot gas responsible for the X-ray emission is needed to account for this emission. Our data does not allow us to discriminate between the various mechanisms proposed in the literature for the soft excess. However, in view of the most recent results obtained with XMM-Newton suggesting that there is much less warm gas in the central regions of clusters than previously believed, it seems likely that this EUV excess is probably of non thermal origin.

Acknowledgements. We are very grateful to M. Traina, from the Observatoire de la Côte d'Azur, for her invaluable help in obtaining the up-to-date version of the multiscale vision model package used throughout the technical part of this paper. We acknowledge discussions with D. Gerbal, G. Lima Neto, G. Mamon and J. Mittaz. We also warmly thank the referee, Jelle Kaastra, for several pertinent suggestions which helped us to improve the paper. Finally, R. Lieu thanks the Institut d'Astrophysique de Paris, CNRS, and the Université Pierre et Marie Curie for their hospitality.

\section{References}

Anscombe, F. J. 1948, Biometrika, 15, 246

Atoyan, A. M., \& Völk, H. J. 2000, ApJ, 535, 45

Berghöfer, T. W., Bowyer, S., \& Korpela, E. 2000a, ApJ, 535, 615

Berghöfer, T. W., Bowyer, S., \& Korpela, E. 2000b, ApJ, 545, 695

Bonamente, M., Lieu, R., \& Mittaz, J. P. D. 2001a, ApJ, 546, 805

Bonamente, M., Lieu, R., \& Mittaz, J. P. D. 2001b, ApJ, 547, L7

Bowyer, S., \& Berghöfer, T. W. 1998, ApJ, 506, 502

Bowyer, S., Lieu, R., \& Mittaz, J. 1998, in The Hot Universe, ed. K. Koyama, S. Kitamoto, \& M. Itoh (Dordrecht: Kluwer), 185 Bowyer, S., Berghöfer, T. W., \& Korpela, E. J. 1999, ApJ, 526, 592

Bowyer, S., Korpela, E. J., \& Berghöfer, T. W. 2001, ApJ, 548, 135

Briel, U. G., \& Henry, J. P. 1996, ApJ, 472, 131

Brunetti, G., Setti, G., Feretti, L., \& Giovannini, G. 2001, MNRAS, 320,365

Dixon, W. V., Sallmen, S., Hurwitz, M., \& Lieu, R. 2001, ApJ, 550, L25

Ensslin, T. A., \& Biermann, P. L. 1998, A\&A, 330, 90

Ensslin, T. A., Lieu, R., \& Biermann, P. L. 1999, A\&A, 344, 409

Fabian, A. C. 1997, Science, 275, 48

Hughes, J. P., Butcher, J. A., Stewart, G. C., \& Tanaka, Y. 1993, ApJ, 404,611

Hwang, Z. 1997, Science, 278, 1917

Hwang, Z., \& Sarazin, C. L. 1998, ApJ, 496, 728

Kaastra, J. S. 1992, An X-ray spectral code for optically thin plasmas, Internal SRON Leiden report v.2.0

Kaastra, J. S., Lieu, R., Mittaz, J. P. D., et al. 1999, ApJ, 519, L119

Kaastra, J. S., Tamura, T., Peterson, J., Bleeker, J., \& Ferrigno, C. 2001, Proc. Symposium New visions of the X-ray Universe in the XMM-Newton and Chandra Era, 26-30 November 2001, ESTEC, The Netherlands

Lieu, R., Mittaz, J. P. D., Bowyer, S., et al. 1996a, Science, 274, 1335

Lieu, R., Mittaz, J. P. D., Bowyer, S., et al. 1996b, ApJ, 458, L5

Lieu, R., Ip, W.-H., Axford, W. I., \& Bonamente, M. 1999a, ApJ, 510, L25

Lieu, R., Bonamente, M., \& Mittaz, J. P. D. 1999b, ApJ, 517, L91

Lieu, R., Bonamente, M., Mittaz, J. P. D., et al. 1999c, ApJ, 527, L77

Lieu, R., Bonamente, M., \& Mittaz, J. P. D. 2000, A\&A, 364, 497

Mewe, R., Gronenschild, E. H. B. M., \& van den Oord, G. H. J. 1985, A\&A, 62, 197

Mallat, S. 1989, IEEE on PAMI, 11(7), 574

Mewe, R., Lemen, J. R., \& van den Oord, G. H. J. 1986, A\&A, 65, 511

Mittaz, J. P. D., Lieu, R., \& Lockman, F. J. 1998, ApJ, 498, L17

Morrison, R., \& McCammon, D. 1983, ApJ, 270, 119

Rué, F., \& Bijaoui, A. 1997, Experimental Astronomy 7, 129

Sarazin, C. L., \& Lieu, R. 1998, ApJ, 494, L177

Siddiqui, H., Stewart, G. C., \& Johnstone, R. M. 1998, A\&A, 334, 71

Snowden, S. L., McCammon, D., Burrows, D. N., \& Mendehall, J. A. 1994, ApJ, 424, 714 